\title{
Early attentional bias for negative words when competition is induced
}

\author{
Ming-Chou Ho ${ }^{1,2} \cdot$ Shuo-Heng $\mathrm{Li}^{3} \cdot$ Su-Ling $\mathrm{Yeh}^{3,4}$
}

Published online: 15 March 2016

(C) The Psychonomic Society, Inc. 2016

\begin{abstract}
Previous research (Zeelenberg, Wagenmakers, \& Rotteveel, 2006) revealed that emotionally meaningful words were identified significantly better than neutral words, with no difference between positive and negative words. Since in that study only a single target word was displayed at a time, we hypothesized that the equivalent performances for positive and negative words were due to a lack of competition. To test this, in our Experiment 1, we replicated Zeelenberg and colleagues' finding, using emotion-laden Chinese words and the identical data-limited method, which measured the accuracy of a briefly shown target. We then introduced competition in Experiment 2 by simultaneously presenting two words during the target frame, and found evidence for an early attentional bias to negative words. In Experiment 3, we confirmed that the bias in Experiment 2 was not due to the inevitable repetition of stimuli. Taken together, these results support our hypothesis that, in the presence of competition, negative words receive attentional priority and consequently have enhanced perceptual representations.
\end{abstract}

Su-Ling Yeh

suling@ntu.edu.tw

1 Department of Psychology, Chung-Shan Medical University, Taichung, Taiwan

2 Clinical Psychological Room, Chung-Shan Medical University Hospital, Taichung, Taiwan

3 Department of Psychology, National Taiwan University, Taipei, Taiwan

4 Neurobiology and Cognitive Science Center, Graduate Institute of Brain and Mind Sciences, National Taiwan University, Taipei, Taiwan
Keywords Attention - Competition · Emotion · Positive · Negative · Data-limited method

By presenting a single word (positive, negative, or neutral) in a target display, Zeelenberg, Wagenmakers, and Rotteveel (2006) reported that perceptual processes are equally enhanced for positive and negative words, relative to neutral words. They used a data-limited method (see also Ho, 2011; Ho \& Atchley, 2009; Norman \& Bobrow, 1975; Prinzmetal, McCool, \& Park, 2005; Santee \& Egeth, 1982), in which a target was presented briefly (set individually, mean duration = $25 \mathrm{~ms}$ ), followed by a mask (300 ms). Immediately after the mask, participants were required, in a two-alternative forced choice (2AFC) procedure, to identify the target from a word pair consisting of the target and a foil word. The emotional valences of the target and foil words were manipulated independently as positive, neutral, or negative, creating a $3 \times 3$ condition matrix. The accuracy of the $2 \mathrm{AFC}$ was used as a measure of processing effectiveness. During the brief target presentation duration, when more information (or better representation quality) are extracted, the accuracy of target identification was higher, indicating better processing effectiveness. Since these results showed that identification accuracy was not biased toward the emotional foil over the neutral target word, Zeelenberg et al. ruled out the possibility of a nonattentional response bias for emotional words. Instead, they argued for enhanced perceptual representations of emotional words and, more relevant to the present study, no difference in perceptual enhancement between positive and negative relative to neutral words.

Earlier studies have also demonstrated a general emotional advantage in attention when presenting a single word at a time using the attentional-blink task. The attentional blink refers to the transient deterioration of the ability to identify the second 
target word after identification of the first target word in a rapid serial visual presentation. However, when the second target word had emotional valence (either positive or negative), it was more often correctly identified than a neutral word, substantially reducing attentional-blink impairment (Anderson, 2005; Keil \& Ihssen, 2004).

When it comes to comparing negative and positive stimuli, negative stimuli seem to have a processing advantage over positive ones. Particularly, studies using multiple stimuli have usually reported faster detection of negative stimuli (a negative emotion advantage). Many studies have adopted stimuli of facial expressions in the visual search task (Frischen, Eastwood, \& Smilek, 2008; Hahn \& Gronlund, 2007; Öhman, Lundqvist, \& Esteves, 2001; Reynolds, Eastwood, Partanen, Frischen, \& Smilek, 2009) and the flanker task (Barratt \& Bundesen, 2012; Fenske \& Eastwood, 2003). For example, Öhman et al. (2001) reported that search speeds were faster when participants had to detect a negative face among positive crowds than when they had to detect a positive face among negative crowds. Studies using the visual probe task (e.g., Bradley, Mogg, \& Lee, 1997; MacLeod, Mathews, \& Tata, 1986) also reported that when two words of different valences appeared simultaneously, anxious participants preferentially attended to the negative word.

However, the mechanism behind this apparent bias is still relatively unknown. On the basis of the pattern that such bias is mostly seen in studies in which multiple stimuli of different emotional valences are presented simultaneously, but not seen in cases without such active competition (e.g., Zeelenberg et al., 2006), we propose that competition in attentional processing between those emotional stimuli of different valences might be a possible explanation for the better performance for negative stimuli over positive ones.

Other than direct competition between stimuli of opposite emotional valences, competitions between emotional valence and other stimulus properties may also exist. Studies using the Stroop task have consistently shown that anxious individuals are not only more likely to have threat-related bias in comparison to their nonanxious counterparts, but are also more prone to color-naming interference when threat-related words are presented (for a meta-analytic study, see Bar-Haim, Lamy, Pergamin, Bakermans-Kranenburg, \& van IJzendoorn, 2007). We suggest that competition could take place in the presence of only one stimulus but between different stimulus properties, as in the Stroop task. Specifically, such competition is created between color naming (the primary task) and the meaning of the stimulus (e.g., negative valence; irrelevant to the primary task). This suggestion is supported by brainimaging studies: The posterior brain regions are activated for processing color and task-irrelevant information (e.g., negative words), and top-down cognitive control is recruited to bias posterior processing toward task-relevant color naming
(Banich et al., 2001). In addition, many brain areas in prefrontal cortex (e.g., posterior-dorsolateral and posterior-inferior prefrontal cortex) are also reported to be involved in such biased processing (Herd, Banich, \& O’Reilly, 2006; Milham, Banich, \& Barad, 2003).

Calvo, Nummenmaa, and Hyönä (2007) provided direct evidence supporting the important role of competition in the attentional processing of emotional information. They presented pleasant or unpleasant scene pictures to participants to examine the attentional processing of emotional scenes. The prime was one (either neutral or emotional) or two (one neutral and one emotional) scene pictures, followed by a probe picture. Participants were instructed to answer whether the content of the probe was identical to that of the prime (single-prime condition) or one of the primes (double-prime condition). They reported attentional facilitation to the emotional scenes only when two prime scenes were presented simultaneously. Given that human processing capacity is limited, competition between multiple stimuli is required for further processing (Awh, Belopolsky, \& Theeuwes, 2012; Desimone \& Duncan, 1995). In such a competitive condition, attention can be allocated to the most salient stimulus and can affect subsequent perceptual processes (e.g., contrast appears higher; Carrasco \& Yeshurun, 2009).

Note that studies using multiple stimuli have usually assumed that attention can affect subsequent perceptual processes, but they have not provided evidence for this assumption. Therefore, in the present study, we adopted the same datalimited method used by Zeelenberg et al. (2006) and examined whether there was an early attentional bias toward negative words. We hypothesized that when multiple emotional words were present, the perceptual representations of emotional words might not be equally enhanced; instead, competition between emotional words could guide earlier attention to them differently (e.g., Bar-Haim et al., 2007; Fenske \& Eastwood, 2003), thereby enhancing their perceptual representations to various extents. More specifically, we hypothesized that in the presence of competition, negative words, in comparison to positive words, can attract attention and thus enhance their own perceptual representations. The theory for the present hypothesis is based on the emotion-specific account of biased competition (e.g., Bar-Haim et al., 2007; Desimone \& Duncan, 1995; Mathews \& Mackintosh, 1998), which hints at the possibility that negative information tends to receive attentional priority, particularly in the presence of competition. When multiple stimuli are present, they compete with each other for limited attentional resources (Desimone \& Duncan, 1995), and a stimulus laden with negative valence better attracts attention for further processing (Bar-Haim et al., 2007; Mathews \& Mackintosh, 1998).

Unlike the Easterbrook hypothesis, which suggests that our attention would narrow with increased arousal, regardless of any positive or negative emotional manipulation 
(Easterbrook, 1959), the present hypothesis emphasizes a negative-emotion advantage in the presence of competition. However, our hypothesis does not contradict the Easterbrook hypothesis, since it appears that arousal and attentional selectivity can be separated (Sørensen \& Barratt, 2014; van Steenbergen, Band, \& Hommel, 2011): Although the amygdala is critical in response to the presence of negative stimuli (LeDoux, 1996), and can trigger a general increase in cortical arousal, resulting in a narrowing of the attentional focus (consistent with the Easterbrook hypothesis), the amygdala can also send a signal of weighted attention reciprocally to visual cortex, causing more-efficient processing of negative stimuli (Sørensen \& Barratt, 2014). Therefore, theoretically, negative words could attract more attention without an increase in arousal level.

Here we chose to use multiple stimuli to test our hypothesis that competition is critical for an early attentional bias to negative words. In Experiment 1, by presenting a Chinese word in the target display, we tested whether we could replicate the results of Zeelenberg et al. (2006). In Experiment 2, we added one additional word to the target display and presented the two words simultaneously to test whether negative words are preferred over positive ones when there is competition between two words in the target display. In Experiment 3, we examined whether repetition of stimuli is critical for the difference in results between Experiments 1 and 2 .

\section{Experiment 1}

In this experiment, we used Chinese words to replicate the work of Zeelenberg et al. (2006), who used Dutch words, for comparison with the two-word condition in the following experiment. We closely followed the method developed by Zeelenberg et al. A target word was presented briefly and then masked. The word-to-mask time intervals were set at a level that maintained participants' accuracy for target identification at about $75 \%$. Immediately after the removal of the mask, two words (one target and one foil) were presented, and participants had to identify which one had been shown previously. If the perceptual representations of emotional words (positive and negative) were enhanced, identification accuracy should be better for emotional than for neutral target words. Alternatively, if better performance was due to a response bias for emotional words, identification accuracy should be better for emotional foils than for neutral target words.

\section{Method}

Participants Thirty undergraduate students from National Taiwan University participated in this study for extra course credit. The sample sizes in the present study were set to be similar to that of Zeelenberg et al. $(2006 ; N=29)$. All were native Mandarin speakers with normal or corrected-to-normal vision. They were naïve about the purpose of the study, and took part in the experiment after informed consent. All experiments in this study followed the human subject ethics guidelines and were approved by the ethics committee of the Department of Psychology at National Taiwan University.

Apparatus and stimuli The stimuli were generated with and controlled by E-Prime software (Schneider, Eschman, \& Zuccolotto, 2002). They were presented on a 21-in. calibrated ViewSonic color monitor (at a refresh rate of $100 \mathrm{~Hz}$ ) in a dimly lighted room. All stimuli were black on a white background and were viewed binocularly with a chin rest at a distance of $50 \mathrm{~cm}$.

Figure 1 shows the sequence of an experimental trial. Each test trial started with the presentation of a column of four line segments $\left(5.06^{\circ}\right.$ in length, separated by a space of $\left.0.06^{\circ}\right)$ at the center of the screen to serve as a warning sign. A rectangle $\left(4.43^{\circ} \times 2.30^{\circ}\right)$ was then presented centrally for $250 \mathrm{~ms}$. This rectangle served as a common-region cue (Palmer, 1992) that supposedly directed participants' attention within this area homogeneously, so as to prevent inappropriate allocation on particular locations of the two-character words (e.g., the location of the upper character). This common-region cue became thicker $\left(4.49^{\circ}\right.$ in height $\times 2.33^{\circ}$ in length $)$ and flashed briefly for $50 \mathrm{~ms}$ to make the cue salient. Following this cue, a twocharacter word $\left(3.90^{\circ}\right.$ in height $\times 2.09^{\circ}$ in length) appeared briefly for $20 \mathrm{~ms}$ in the target display. Each character was $2.09^{\circ}$ in height and $1.90^{\circ}$ in length. Each word consisted of one character above, and one below (i.e., a vertically arranged two-character Chinese word). After this word was presented, a mask $\left(5.06^{\circ} \times 2.39^{\circ}\right)$ consisting of four random-stroke patterns (each $1.24^{\circ} \times 2.39^{\circ}$, separated by a space of $1.07^{\circ}$ ) immediately covered the word. Finally, in the test display, two vertically presented words (one target and one foil) were presented at the left and right sides of the display. This display would remain until the participant made a two-alternative forced choice (2AFC) response as to which one (left or right) was the target. In the test display, the horizontal distance between the center of the word and the center of the display was $4.56^{\circ}$. Both the target and the foil were two-character words in an identical font (Shi-Ming font).

A set of 144 words was selected on the basis of subjective ratings by another group of undergraduate students at the National Taiwan University $(N=30)$ on the emotional valence of the words using a valence scale of 1 (most negative) to 7 (most positive) and an arousal scale of 1 (most weak) to 7 (most strong). The two scales were rated separately. The rating results showed main effects of valence $[F(2,58)=115.316, p$ $\left.<.001, \eta_{\mathrm{p}}{ }^{2}=.799\right]$ and arousal $[F(2,58)=30.149, p<.001$, $\left.\eta_{\mathrm{p}}{ }^{2}=.510\right]$. In the present study, all the values reported from the follow-up tests are based on $t$ tests. Fisher's least significant difference test was used to control the familywise alpha 


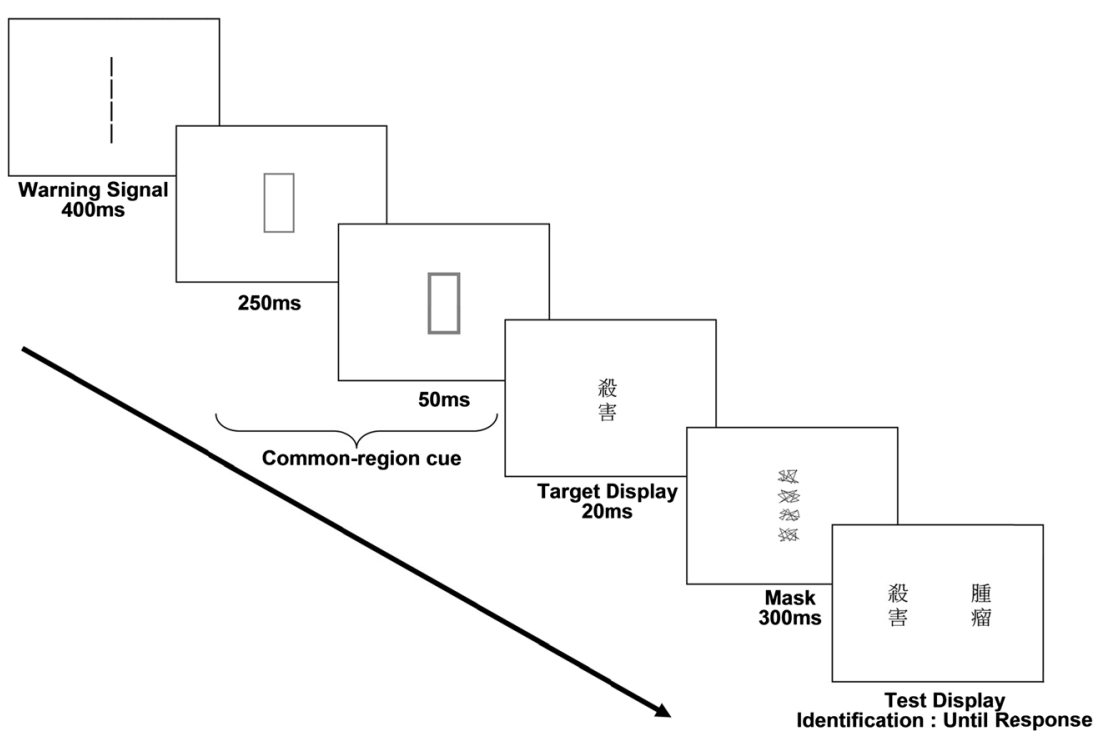

Fig. 1 Sample procedure in Experiment 1. A target word was shown on the target display, followed by two words (the target and the foil) in the test display. Both the target and foils words were two-character Chinese words. The task was to recognize the target word in the test display. In this

value at .05 . The valence scores among the negative words (mean score $=2.6)$, neutral words (mean score $=4.0$ ), and positive words (mean score $=5.3$ ) differed significantly (all $p$ s $<.001$ ). Both positive (mean score $=4.3$ ) and negative (mean score $=4.5$ ) words had higher arousal scores than did neutral ones (mean score $=2.9$; all $p$ s $<.001$ ), but there was no difference between the arousal scores for positive and negative words $(p=.472)$. Since arousal may direct attention to emotional events (e.g., Anderson, 2005; Arnell, Killman, \& Fijavz, 2007; Most, Smith, Cooter, Levy, \& Zald, 2007; Schimmack \& Derryberry, 2005; Vogt, De Houwer, Koster, Van Damme, \& Crombez, 2008), the levels of arousal of positive and negative words were controlled to be similar.

As to the characteristics of the three groups of words used, there was no effect of word frequency $[F(2,94)=0.404, p=$ $\left..669, \eta_{\mathrm{p}}{ }^{2}=.009\right]$, on the basis of the Word Frequency database published by the Ministry of Education in Taiwan (National Languages Committee, 1997). Since the word frequencies between three groups of words were controlled, this excluded the possibility that the word frequency, but not the emotional valence of the words, was what affected the results. A oneway analysis of variance (ANOVA; Emotion Valence: negative, neutral, and positive) on stroke counts showed no main effect of emotional valence $\left[F(2,94)=0.116, p=.891, \eta_{\mathrm{p}}{ }^{2}=\right.$ $.002]$, which helped to rule out the possibility that the bottomup strokes of two-character words affected the results.

Design Each of the emotional valence (negative, neutral, and positive) conditions contained 48 emotion-laden words (e.g., murder). The 48 words were divided randomly into six sets of eight words each, with three sets serving as targets and the remaining three sets as foils. The three target sets were paired example, the target (meaning murder) presented in the target display is subsequently shown in the test display on the left side, and the foil word (meaning tumor) is on the right side

with negative, positive, and neutral foils, respectively. Also, the three foil sets were paired with negative, positive, and neutral targets, respectively. Therefore, a target from one of the three emotional valences (negative, neutral, and positive) was paired with a foil from one of the three valences, giving rise to nine $(3 \times 3)$ conditions. Since each set consisted of eight words, each condition included eight target-foil pairs, making 72 trials in total $(9 \times 8)$. Finally, to ensure that word pairs were constructed with words from all valences, the six sets of words in each valence were rotated to serve as targets and foils, rendering six combinations, each assigned to a group of five participants.

Procedure The experiment consisted of three phases in sequence: instruction, practice, and experiment. In all three phases, each trial began with the warning signal for $400 \mathrm{~ms}$. An outline square was presented for $250 \mathrm{~ms}$ afterwards, followed by a 50-ms thickening cue, and then one target word was presented. In the instruction phase (two trials), each word was presented on the screen for $200 \mathrm{~ms}$ to familiarize the participant with the task. This was followed by a practice phase (48 trials) in which all words in the target display were presented for $20 \mathrm{~ms}$. This duration was established in a pilot study with six undergraduate students from the National Taiwan University in order to maintain the participants' accuracy at about $75 \%$. Indeed, in the present study, the average accuracy rates across Experiments 1-3 were about $75 \%$ ( $74 \%, 74 \%$, and $76 \%$, respectively). Hence, it appears that the adopted presentation duration was appropriate. In the experiment phase, the target presentation time was the same one used in the practice phase. It was masked for $300 \mathrm{~ms}$, and then entered into the test display until the participant had pressed 
the key to indicate which one (left or right) was the target. Participants were instructed to press the " $\mathrm{z}$ " key if the target was on the left side, and the "/" key if the target was on the right side. After completion of the $2 \mathrm{AFC}$ task, participants were asked to rate the emotionality of the words used in the experiment. The words were rated on the same 7-point scale. This manipulation check was done to ensure that the participants who took part in the experiment rated the three valences of the words (positive, neutral, and negative) similarly to the prerating task conducted with a different group of participants.

\section{Results}

Manipulation check The mean emotional valences of the participants' ratings of the words differed significantly among the three groups of words $[F(2,58)=579.328, p$ $\left.<.001, \eta_{\mathrm{p}}{ }^{2}=.952\right]$. Negative words had lower rating scores (mean score $=2.5$ ) than did neutral words (mean score $=4.1$, which had lower scores than positive words (mean score $=5.5$; all $p$ s $<.001$ ). As with the ratings by another group of participants for the selection of the stimulus materials, the ratings by the same group of participants who completed the experiment confirmed the validity of our choice of words: These words were indeed perceived as having different emotional valences.

Target identification Figure 2 a shows the correctidentification rates for the target. A two-way repeated measure ANOVA with the factors Target Emotion (negative, neutral, or positive) and Foil Emotion (negative, neutral, or positive) was conducted on the target identification accuracies (Table 1). We found a main effect of target emotion $[F(2,58)=4.858, p=$ $\left..011, \eta_{\mathrm{p}}{ }^{2}=.143\right]$, but no main effect of foil emotion, and no interaction between them $(p s>.2)$. The performances at recognizing positive targets (mean correct rate $=76.3 \%$ ) and
Table 1 Mean accuracy (\%) of target identification in Experiment 1 as a function of the emotional valences of the target and competitor

\begin{tabular}{llll}
\hline Target & \multicolumn{2}{l}{ Competitor } & \\
\cline { 2 - 4 } & Negative & Neutral & Positive \\
\hline Negative & $79.0(3.2)$ & $75,7(3.3)$ & $73.2(3.4)$ \\
Neutral & $66.5(3.8)$ & $74.5(3.6)$ & $71.1(3.7)$ \\
Positive & $75.2(3.0)$ & $76.5(2.9)$ & $75.6(3.1)$ \\
\hline
\end{tabular}

Numbers in parentheses are standard errors of the means

negative targets (mean correct rate $=76.1 \%$ ) were better than performance at recognizing neutral targets (mean correct rate $=70.6 \%)\left[t(29)=2.812, p=.009, \eta_{\mathrm{p}}^{2}=.214\right.$, and $t(29)=$ $3.025, p=.005, \eta_{\mathrm{p}}{ }^{2}=.240$, respectively]. There was no difference between the identification rates of positive targets and negative targets $\left[t(29)=0.075, p=.941, \eta_{\mathrm{p}}^{2}<.001\right]$.

We also conducted the same ANOVA on the response times (RTs), yielding neither any main effects nor an interaction between the factors (all $p \mathrm{~s}>.30$ ).

\section{Discussion}

In this experiment, we found that when a single Chinese emotional word was presented briefly, it was recognized more accurately than a neutral word; however, no difference in identification accuracies was apparent between negative and positive words. This result was consistent with what was found using Dutch words (Zeelenberg et al., 2006), indicating the sensitivity of our measure for probing this issue and the generalizability of the results to a script very different from alphabetic systems. Next we presented two words simultaneously in the target display, to test the hypothesis that when competition
(A)

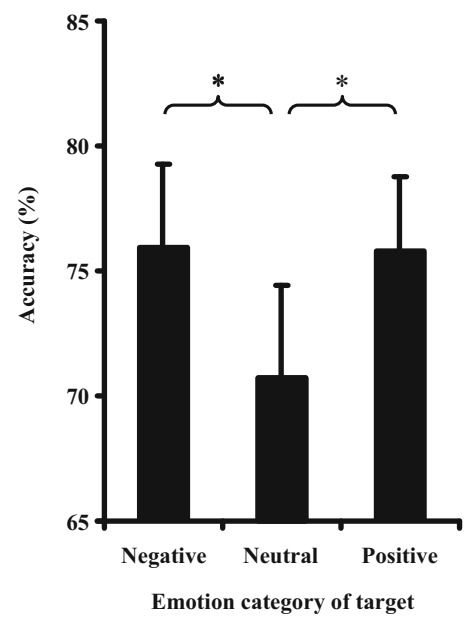

(B)

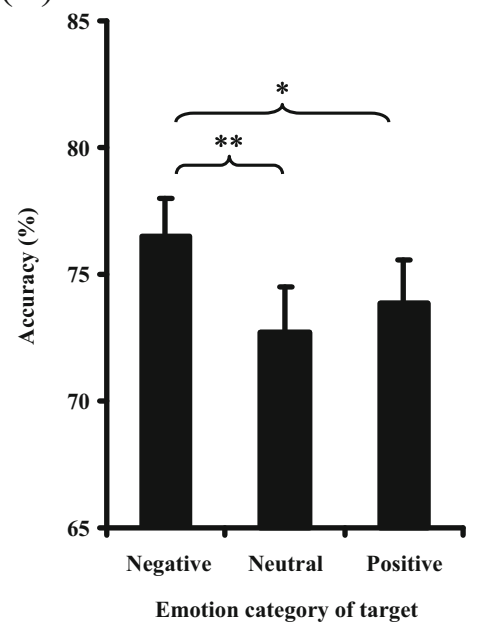

(C)

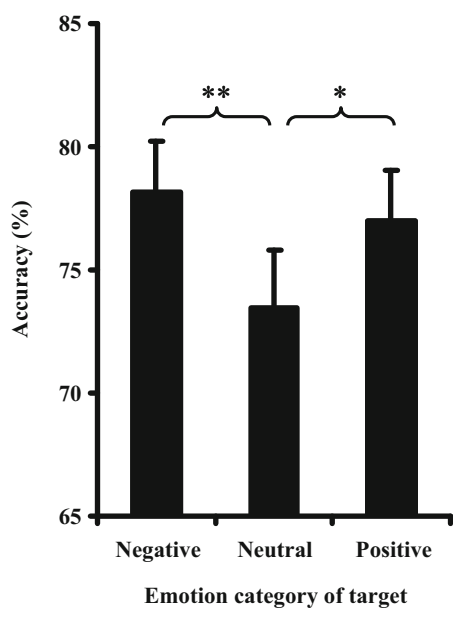

Fig. 2 Mean accuracies of target identification in Experiments 1 (a), 2 (b), and 3 (c). Error bars represent one standard error of the mean 
for attentional resource was induced by presenting multiple words in the display, negative words would be preferred over positive ones in this competitive situation.

\section{Experiment 2}

The design and stimuli were kept as similar as possible to those of Experiment 1, except for the following modifications (Fig. 3). Two words - one target word and one competitorwere presented simultaneously in the target display. The target and its competitor were each selected from one of the three emotional valences (negative, positive, and neutral), creating nine $(3 \times 3)$ conditions. Namely, the target and competitor could be from the same or from different emotional valence conditions. In the test display, the target was presented again, along with a different word as the foil that had the same valence as the target. We matched the valences of the target and foil for the following reasons. First, if the target and foil did not match in emotional valence, this would create two competitions (target vs. competitor during target presentation, and target vs. foil in the test display), thus complicating explanation of the results. Also, this double-competition design would enormously increase the number of trials. Finally, to answer our question (whether there is an early attentional bias toward negative words), it was more appropriate to introduce competition only during the target presentation, since it was the display that was manipulated as one versus two words to see the effect of competition (without vs. with competition) in Experiments 1 and 2.
The participants' task was again to indicate which word in the test display had appeared in the target display, where this time two words were presented together to induce a competition for attention. We predicted that, under competition, participants would prefer negative over positive ones.

\section{Method}

Participants Thirty undergraduate students at the National Taiwan University participated in this study for extra course credits. All were native Mandarin speakers with normal or corrected-to-normal vision. They were naïve about the purpose of the study and gave informed consent.

Design and stimuli In the target display, one word was located $1^{\circ}$ (center-to-center distance) to the left of the central location, and the other was located $1^{\circ}$ to the right. Both the target and the competitor were located $1^{\circ}$ (center-to-center distance) to the left and right of the central location. The locations of target and competitor were randomized and equally probable to occur on the left and the right. The warning signal consisted of two columns of four line segments $\left(5.06^{\circ}\right.$ in length, separated by a space of $0.06^{\circ}$ ), and the masks were two columns of random-stroke patterns. Both warning signal and masks were located $1^{\circ}$ (center-to-center distance) to the left and right of the central location. The common-region cue was changed to $4.43^{\circ} \times 4.60^{\circ}$, and the thickening cue was changed to $4.49^{\circ}$ $\times 4.66^{\circ}$.

The whole experiment consisted of two instruction trials, 48 practice trials, and 288 formal trials. This experiment adopted the same set of words used in Experiment 1. Forty-

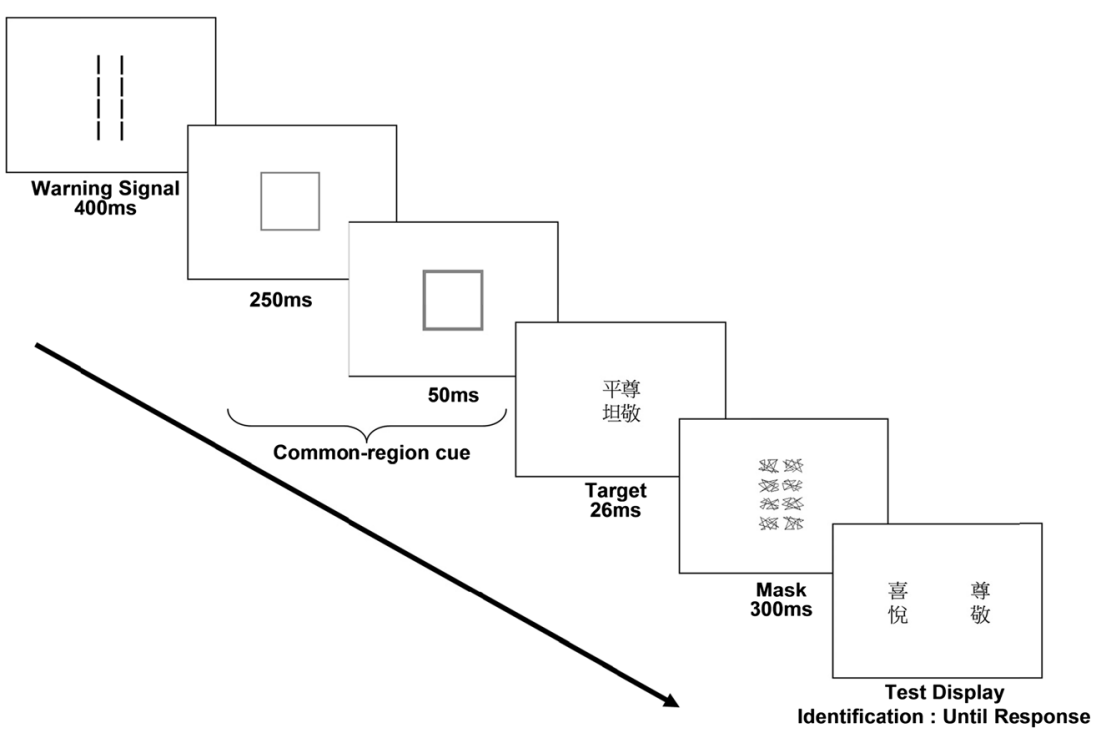

Fig. 3 Sample procedure in Experiment 2. A target (in this example it means respect, on the right), and a competitor (meaning flat, on the left) were presented simultaneously, followed by a test display in which a target identification task was required in which the participants had to indicate which word had appeared previously. In the test display, the target (respect, on the right) and the foil (joy, on the left) were different words with the same emotional valence (i.e., both are "positive" words) 
eight words of each emotional valence were divided randomly into six sets of eight words each. Among the six sets, three served as targets, and the other three were repeated once, to serve as both competitors and foils. The three target sets were paired with negative, positive, and neutral competitors, respectively. Also, the three competitor sets and three foil sets were paired with negative, positive, and neutral targets, respectively. Thus, a target from one of the three emotional valences (negative, positive, and neutral) was paired with a competitor from one of the three valences, giving rise to nine $(3 \times 3)$ conditions. Since each set consisted of eight words, each of the nine conditions had eight target-foil pairs, making a total of 72 trials in a block $(8 \times 9)$. This block was repeated four times, resulting in 288 trials in total $(72 \times 4)$. Finally, to ensure the construction of word pairs with words from all valences, the six sets of words for each valence were rotated to serve as targets and foils, rendering six combinations, each being assigned to a group of six participants.

Procedure The procedure was similar to that of Experiment 1, except that the target and competitor were presented for $26 \mathrm{~ms}$, which was based on a pilot study showing that this duration was appropriate for maintaining the mean accuracy at $75 \%$.

\section{Results}

Manipulation check We found a significant difference in the ratings of emotional valence $\left[F(2,58)=823.837, p<.001, \eta_{\mathrm{p}}{ }^{2}\right.$ $=.966]$. Negative words had lower rating scores (mean score $=2.3$ ) than did neutral words (mean score $=4.1$ ), which had lower scores than positive words (mean score $=5.7)(p s<$ .001 ). This indicates that for the present group of participants, the words selected from one emotional valence indeed differed from those of other valences, and the direction was as predicted in terms of emotional valence.

Target identification We conducted a two-way repeated measure ANOVA with the factors Target Emotion (negative, neutral, or positive) and Competitor Emotion (negative, neutral, or positive) on identification accuracies. The main effect of the target emotion $\left[F(2,58)=6.235, p=.004, \eta_{\mathrm{p}}{ }^{2}=.177\right]$ was significant, but that of the competitor emotion was not $[F(2$, $\left.58)=2.610, p=.082, \eta_{\mathrm{p}}{ }^{2}=.083\right]$. Further tests indicated that negative target words contributed to higher accuracy than did neutral and positive target words (Fig. 2b). More importantly, we obtained an interaction between target emotion and competitor emotion $\left[F(4,116)=2.975, p=.022, \eta_{\mathrm{p}}{ }^{2}=.093\right]$. Further analysis of this interaction is described below.

First, when paired with a negative competitor, identification of a negative target (mean accuracy rate $=76.8 \%$ ) was better than identification of a neutral target (mean accuracy rate $=71.7 \%$ ) and a positive target (mean accuracy rate $=$
$69.7 \%)\left[F(2,58)=4.651, p=.013, \eta_{\mathrm{p}}{ }^{2}=.138\right]($ Table 2$)$. For clarity, we denote the above finding as $\mathrm{T}_{-} \mathrm{C}_{-}>\mathrm{T}_{\mathrm{n}} \mathrm{C}_{-}$and $\mathrm{T}$ $\mathrm{C}_{-}>\mathrm{T}_{+} \mathrm{C}_{-}$, where " $\mathrm{T}$ " refers to the target, " $\mathrm{C}$ " refers to the competitor, "+" refers to a positive emotion, "-" refers to a negative emotion, and " $n$ " refers to neutral. We adopt this format for expressions hereafter.

Also, when paired with a positive competitor, identification of the negative target (mean accuracy rate $=79.0 \%$ ) was better than identification of a neutral target (mean accuracy rate $=72.4 \%$ ) or a positive target (mean accuracy rate $=$ $73.5 \%)\left[F(2,58)=6.661, p=.002, \eta_{\mathrm{p}}^{2}=.187\right]$; that is, $\mathrm{T}$ $\mathrm{C}_{+}>\mathrm{T}_{\mathrm{n}} \mathrm{C}_{+}$and $\mathrm{T}_{-} \mathrm{C}_{+}>\mathrm{T}_{+} \mathrm{C}_{+}$.

Finally, we found that when the target was a positive word, identification of this word was better when it was coupled with a neutral competitor (mean accuracy rate $=76.8 \%$ ) than when it was coupled with a negative competitor (mean accuracy rate $\left.=69.7 \%)\left[F(1,29)=11.169, p=.002, \eta_{\mathrm{p}}^{2}=.278\right)\right]$ - that is, $\mathrm{T}_{+} \mathrm{C}_{\mathrm{n}}>\mathrm{T}_{+} \mathrm{C}_{-}$. No other difference was found by our postanalysis here.

The same ANOVA were also conducted on the RTs, and neither any main effects nor an interaction emerged (all $p \mathrm{~s}>.10$ ).

\section{Discussion}

The present results differed from those in Experiment 1 and Zeelenberg et al. (2006), in which only one target was presented. In Experiment 1 and Zeelenberg et al., negative and positive words were found to be enhanced equivalently. However, when two words were presented at the same time in this experiment, we found that, in general, negative words were recognized better than positive words, supporting our hypothesis that negative words in a competitive condition could attract attention better than neutral and positive words, so as to enhance their perceptual representation. Our finding was also reaffirmed in further analyses. First, negative targets were recognized better than neutral and positive targets when the competitor was either negative ( $\mathrm{T}_{-} \mathrm{C}_{-}>\mathrm{T}_{\mathrm{n}} \mathrm{C}_{-}$and $\mathrm{T}_{-} \mathrm{C}_{-}>$ $\mathrm{T}_{+} \mathrm{C}_{-}$) or positive $\left(\mathrm{T}_{-} \mathrm{C}_{+}>\mathrm{T}_{\mathrm{n}} \mathrm{C}_{+}\right.$and $\mathrm{T}_{-} \mathrm{C}_{+}>\mathrm{T}_{+} \mathrm{C}_{+}$). Second, the prediction that negative words in a competitive condition could attract attention better was also supported in a further

Table 2 Mean accuracy (\%) of target identification in Experiment 2 as a function of the emotional valences of the target and competitor

\begin{tabular}{llll}
\hline Target & \multicolumn{2}{l}{ Competitor } & \\
\cline { 2 - 4 } & Negative & Neutral & Positive \\
\hline Negative & $76.8(1.6)$ & $75.2(1.7)$ & $79.0(1.6)$ \\
Neutral & $71.7(2.3)$ & $74.3(1.9)$ & $72.4(1.8)$ \\
Positive & $69.7(1.8)$ & $76.8(1.5)$ & $73.5(1.4)$ \\
\hline
\end{tabular}

Numbers in parentheses are standard errors of the means 
analysis showing that a negative competitor could direct attention to itself, worsening the identification of a positive target $\left(\mathrm{T}_{+} \mathrm{C}_{\mathrm{n}}>\mathrm{T}_{+} \mathrm{C}_{-}\right)$.

\section{Experiment 3}

The results from the previous two experiments suggested that competition is critical (competition was created in Exp. 2, but not in Exp. 1) for better processing of negative than of positive words. However, we cannot rule out the possibility that the difference that we observed was associated with repetition, because we had presented the stimuli four times in Experiment 2, but not in Experiment 1. Thus, it may be that the repetition of stimuli, rather than the competition between stimuli, caused better processing of negative words. To examine this possibility, we replicated Experiment 1 here, but repeated the stimuli four times, just like those in Experiment 2. If repetition of stimuli (rather than competition) is critical, better processing of negative than of positive words should be observed, as in Experiment 2.

\section{Method}

Participants Thirty undergraduate students at the National Taiwan University participated in this study for extra course credits. All were native Mandarin speakers with normal or corrected-to-normal vision. They were naïve about the purpose of the study and gave informed consent.

Design, stimuli, and procedure The design of Experiment 3 was similar to that of Experiment 1, except that the stimuli were repeated four times (288 formal trials). The stimuli and procedure were the same as those in Experiment 1.

\section{Results}

Manipulation check The mean emotional valences of participants' ratings of the words differed significantly among the three groups of words $\left[F(2,58)=488.883, p<.001, \eta_{\mathrm{p}}{ }^{2}=\right.$ .944]. Negative words had lower rating scores (mean score $=$ 2.3) than did neutral words (mean score $=4.1$ ), which had lower scores than positive words (mean score $=5.7$; all $p \mathrm{~s}<$ $.001)$. As with the ratings by another group of participants for the selection of the stimulus materials, the ratings by the same group of participants who completed the experiment confirmed the validity of our choice of words: These words were indeed perceived as having different emotion valences.

Target identification Figure 2c shows the correctidentification rates of the target. A two-way repeated measure ANOVA with the factors Target Emotion (negative, neutral, or positive) and Foil Emotion (negative, neutral, or positive) was conducted on the target identification accuracies (Table 3). We found a main effect of target emotion $[F(2,58)=8.961, p<$ $\left..001, \eta_{\mathrm{p}}{ }^{2}=.236\right]$, but no main effect of foil emotion, and no interaction between them $(p s>.2)$. The performances at recognizing positive targets (mean correct rate $=77.0 \%$ ) and negative targets (mean correct rate $=78.1 \%$ ) were better than performance at recognizing neutral targets (mean correct rate $=73.4 \%)\left[t(29)=3.006, p=.005, \eta_{\mathrm{p}}{ }^{2}=.238\right.$, and $t(29)=$ $4.325, p<.001, \eta_{\mathrm{p}}{ }^{2}=.392$, respectively]. No difference was apparent between the identification rates for positive and negative targets $\left[t(29)=0.984, p=.333, \eta_{\mathrm{p}}{ }^{2}=.032\right]$.

The same ANOVA on the RTs showed no main effects and no interaction between the factors (all $p \mathrm{~s}>.10$ ).

\section{Discussion}

The present results were similar to those in Experiment 1, revealing that negative and positive words were enhanced equivalently. This suggests that repetition of stimuli was not critical for the better processing of negative words observed in Experiment 2. Otherwise, the present results should have been similar to those in Experiment 2, because stimuli were repeated four times in both experiments. The results also indicated that when competition between stimuli was not created in Experiments 1 and 3, the identification accuracies for negative and positive words were comparable, no matter whether the stimuli were repeated (Exp. 3) or not (Exp. 1).

In sum, we reported that better processing for negative than for positive words could be revealed in the face of competition. We suggested a possibility that negative words might direct attention in competitive conditions, thereby enhancing the perception of these words.

\section{General discussion}

We examined whether competition between emotional words can direct early attention differently to enhance the perceptual representations of these words. In Experiment 1, using Chinese words, we replicated the results of a study using Dutch by Zeelenberg et al. (2006) that had demonstrated that emotional words were recognized better than neutral words, with no difference between the identification of positive and

Table 3 Mean accuracy (\%) of target identification in Experiment 3 as a function of the emotional valences of the target and competitor

\begin{tabular}{llll}
\hline \multicolumn{5}{c}{ Competitor } & \\
\hline Target & Negative & Neutral & Positive \\
Negative & $76.8(1.9)$ & $79.4(2.3)$ & $78.3(2.1)$ \\
Neutral & $72.9(2.5)$ & $74.8(2.2)$ & $72.6(2.5)$ \\
Positive & $75.6(2.0)$ & $77.0(2.4)$ & $78.3(1.9)$ \\
\hline
\end{tabular}

Numbers in parentheses are standard errors of the means 
negative words. In this part of the study, only a single Chinese word was presented on each trial in the target display. In Experiment 2, competition was introduced by simultaneously presenting two words during the target phase; we found that identification accuracy was modulated by the emotional valences of both the target and competitor words. In Experiment 3 , we ruled out the possibility that repetition of the stimuli contributed to the difference in results in Experiments 1 and 2. The present results thus supported our hypothesis that, in the presence of competition, negative words receive attentional priority, and their perceptual representations are enhanced (negative emotion advantage).

\section{A closer look at the result patterns in Experiment 2}

For the critical manipulation of introducing multiple words in the target display in Experiment 2, scrutiny of the result pattern may lead one to suspect that stronger support should come from the main effect of the competitor, particularly, for all three target types: $\mathrm{C}_{+}>\mathrm{C}_{-}$and $\mathrm{C}_{\mathrm{n}}>\mathrm{C}_{-}$. In fact, we were less likely to obtain a main effect of competitor in the present study. When both the target and competitor were negative ( $T$ _C_-), they could each attract attention on themselves, thereby facilitating word identification (higher accuracy rate). Therefore, the main effect of competitor (or the lowest accuracy in the condition of negative competitor, regardless of target types) was less likely to be significant in the present case.

One may also insist that the patterns of the target by competitor interactions do not seem to be a sufficiently strong support for our hypothesis. For example, when the target was neutral, the accuracy rates for the competitor across all three emotions were comparable $\left(\mathrm{T}_{\mathrm{n}} \mathrm{C}_{-}=\mathrm{T}_{\mathrm{n}} \mathrm{C}_{\mathrm{n}}=\mathrm{T}_{\mathrm{n}} \mathrm{C}_{+}\right)$. Similarly, when the competitor was neutral, the accuracy rates for the target across all three emotions were comparable $\left(T_{-} C_{n}\right.$ $=T_{n} C_{n}=T_{+} C_{n}$ ). It was also suggested to us that if the competitor were neutral, the accuracy rates of positive and negative targets should be greater than that of neutral target, as in the one-stimulus case (Exp. 1). We recognize that these findings may not be the strongest support for our hypothesis, because we had predicted that when the target (or competitor) was neutral, the accuracy rate of the negative competitor (or negative target) should yield to the most extreme results. However, our hypothesis does not argue that, when multiple stimuli are present, competition should "always" take place. Rather, the present data suggest that, although there is indeed competition when both the target and competitor words are emotional, such competition may attenuate if only one of the two words is emotional. Hence, the seeming absence of a difference in accuracy rates across different emotions may have been the result of decreased competition when only one of the two stimuli was emotional. In other words, lesser between-stimulus competition may have rendered our measure insensitive to the difference between positive and negative emotions we had predicted.

This notion of lesser competition can also be applied to other descriptive statistics that seemed inconsistent with our hypothesis. Individually, these were that (1) negative target accuracy was lowest when the target was presented with a neutral competitor (lowest $T_{-} C_{n}$ accuracy), and (2) positive target accuracy was higher than negative target accuracy when targets were presented with a neutral competitor $\left(\mathrm{T}_{+} \mathrm{C}_{\mathrm{n}}>\mathrm{T}\right.$ $\left.{ }_{-} C_{n}\right)$. Although these differences were not statistically significant, this peculiar pattern of results may have been due to lesser between-stimulus competition, so that our measure could have become insensitive to meaningful differences.

\section{Advantages of the data-limited method}

In Experiments 1 to 3, we used the data-limited method in conjunction with the $2 \mathrm{AFC}$ procedure, in a manner similar to that used by Zeelenberg et al. (2006), because of several advantages it has. First, the data-limited method has been demonstrated to successfully exclude a possible confound of response bias (e.g., Prinzmetal, McCool, \& Park, 2005; Santee \& Egeth, 1982). Zeelenberg et al.'s results showing that identification accuracy was not biased toward emotional foils further ensured that emotional words lead to perceptual enhancement but not response bias. Second, because the stimuli were always presented at the same locations and participants knew their locations in advance, another possible confound - a sudden onset, which is known to capture attention regardless of its content (Yantis, 1993; Yeh \& Liao, 2008, 2010) — can also be excluded. With these advantages, we suggest that the present results indeed reflect enhancement of the perceptual representations of emotional stimuli, ruling out the possibility of response bias or attentional capture.

\section{Selection in memory representations}

It could be argued that the identification performance in the present study may reflect selection occurring in the remembered representations, rather than in the perceptual representations. That is, the qualities of the perceptual representations were the same, and participants' performance was dependent on the selection process in short-term memory. However, we suggest that the selection in remembered representations could not exclusively account for the results in the data-limited method. In this method, there is no retention period (usually about 1,000 to 2,500 ms) during which the to-be-remembered stimuli are removed from the display and participants are required to maintain the stimuli in short-term memory for later recall. Instead, the stimuli in the present data-limited experiment were presented very briefly (20 and $26 \mathrm{~ms}$ in Exps. 1 and 2 , respectively), and participants made responses immediately after these stimuli were masked. Therefore, participants 
should have relied less on the memory representations when reporting the target. Moreover, taking advantage of the datalimited method, the present study links early attentional bias to subsequent perception. This is important because many studies simply assume that attention can affect perception, but few studies have directly tested this hypothesis, as we did here.

\section{The present design and visual search}

The design of Experiments 1 and 2, in which the targets (and the competitors in Exp. 2) were not defined until the alternatives were presented in the identification task, differs from those in previous studies, in which the target was predefined (e.g., a visual search task). Our design was more advantageous, because a predefined target might bias emotional competition between words, which might confound the major goal (emotional competition) of this study. For example, a predefined neutral target can attract attention and possibly override the attentional attraction by a negative competitor. This could reduce or even eliminate emotion-modulated attentional processing under the situation of competition.

\section{The present findings and Calvo et al. (2007)}

Our conclusion that negative stimuli can attract attention when competition among multiple stimuli is introduced is consistent with the competition hypothesis proposed by Weierich, Treat, and Hollingworth (2008). They suggested that, in the presence of stimulus competition (e.g., a visual search task), participants can detect negative information (e.g., threat) more efficiently (see also Yiend, 2010), yet they did not test this directly.

Consistent with our result in Experiment 2, Calvo, Nummenmaa, and Hyönä (2007) provided evidence supporting the role of competition in attentional processing of emotional information. This suggests that when multiple stimuli competed for attentional resources, emotional, rather than neutral, stimuli were preferentially attended and efficiently encoded. However, Calvo et al. did not reveal different processing between pleasant and unpleasant scenes. In their two-prime condition, they always paired the emotional scene with a neutral one. Similarly, in the present Experiment 2, we reported that when the competitor was neutral, no identification differences emerged across different target emotions (i.e., $\mathrm{T}_{-} \mathrm{C}_{\mathrm{n}}=\mathrm{T}_{\mathrm{n}} \mathrm{C}_{\mathrm{n}}=\mathrm{T}_{+} \mathrm{C}_{\mathrm{n}}$ ). The same phenomenon was also observed when a neutral target was paired with competitors with all three emotions $\left(\mathrm{T}_{\mathrm{n}} \mathrm{C}_{-}=\mathrm{T}_{\mathrm{n}} \mathrm{C}_{\mathrm{n}}=\mathrm{T}_{\mathrm{n}} \mathrm{C}_{+}\right)$. Alternatively, when the competitor was negative or positive, the emotionality of the target became critical to target identification. It is thus possible that the previously introduced idea of lesser between-stimulus competition when only one stimulus is emotional can also account for Calvo et al.'s finding.
The result derived from Calvo et al. (2007)'s singlestimulus case (i.e., when one prime was presented, emotional and neutral primes were processed equivalently) was inconsistent with the single-stimulus case in the present study (Exp. 1) and with Zeelenberg et al. (2006), in which a single emotional word was identified better than a neutral one. This inconsistency has been found in many tasks with only one stimulus in the target display. For example, some studies (e.g., those using a cueing task) have shown equivalent attentional processing of emotional and neutral stimuli (e.g., Buckley, Blanchard, \& Hickling, 2002; Dalgleish et al., 2003; Lim \& Kim, 2004; Mogg, Philippot, \& Bradley, 2004); however, other studies (e.g., those using the data-limited approach, similar to the present study) have reported preferential attentional processing of emotional stimuli (e.g., Dijksterhuis \& Aarts, 2003; Gaillard, Del Cul, Naccache, Vinckier, Cohen, \& Dehaene, 2006). It is unclear what causes this inconsistency in the single-stimulus case. One possibility may be the location of the single stimulus (e.g., foveal vs. parafoveal). In the present study and that of Zeelenberg et al., the stimuli were presented foveally, whereas Calvo et al. presented them parafoveally. It is possible that foveal stimuli can gain more semantic processing than parafoveal stimuli (Calvo \& Castillo, 2005). Therefore, for a foveally presented stimulus whose semantic contents have been processed, an emotional stimulus, rather than a neutral one, can have a better chance of attracting attention and being identified (e.g., Zeelenberg et al., 2006, and the present Exp. 1).

\section{Emotion-specific accounts}

The emotion-specific accounts of both the biased-competition approach (e.g., Bar-Haim et al., 2007; Desimone \& Duncan, 1995; Mathews \& Mackintosh, 1998) and the extent of attentional focus (e.g., Fenske \& Eastwood, 2003; Rowe, Hirsh, \& Anderson, 2007) can together explain our finding of emotionmodulated attentional processing in the presence of multiple stimuli. The former (the biased-competition approach) emphasizes how attentional priority is assigned to different stimuli, and the latter (the extent of attentional focus) emphasizes how attention is allocated in terms of emotional valences.

Because of the limited capacity of our cognitive system, competition for attention occurs between representations of stimuli. Desimone and Duncan (1995) proposed that both bottom-up and top-down factors influence the relative activations of these representations, therefore biasing the competition. In the present study, the stroke counts between negative, neutral, and positive words were controlled to be constant between conditions, thus reducing the influence of bottomup factors from the perceptual representations. That is, the perceptual representations of the words in the present study were less likely to guide attention. Instead, we suggested that the top-down factor of semantic representations (Cave \& 
Batty, 2006) played an important role in the biased competition explored here.

The semantic representations of words are quickly activated (Cave \& Batty, 2006), even without awareness (Yang \& Yeh, 2011; Yeh, He, \& Cavanagh, 2012) and evaluated in terms of the strength of their emotional valences (e.g., threat; LeDoux, 1996; Mathews \& Mackintosh, 1998). Words associated with negative emotion tend to receive attentional priority (Mathews \& Mackintosh, 1998). In addition, when a negative word is attended to, attentional focus is concentrated (Fenske \& Eastwood, 2003), leaving fewer resources for the surrounding nonnegative words. This is similar to a phenomenon called the weapon focus effect (Loftus, 1979; Pickel, 2007; Steblay, 1992), which refers to a constricted attentional focus on a weapon (e.g., a gun), decreasing the ability to process the surrounding details (e.g., the details about the person holding the gun or a crime scene).

This emotion-specific account may explain our results. In the presence of competition, attention can be guided to the negative target (rather than a nonnegative target), causing better identification of this target (e.g., $\mathrm{T}_{-} \mathrm{C}_{+}>\mathrm{T}_{\mathrm{n}} \mathrm{C}_{+}$and $\mathrm{T}_{-} \mathrm{C}_{+}>$ $\mathrm{T}_{+} \mathrm{C}_{+}$). When more than one negative word is presented, attention can concentrate on these negative words even more, thereby facilitating word identification. Therefore, in our case, the identification of a negative word presented in a pair of negative words was better than that of a nonnegative (neutral or positive) word flanked by a negative word - that is, $\mathrm{T}_{-} \mathrm{C}_{-}>$ $\mathrm{T}_{\mathrm{n}} \mathrm{C}_{-}$and $\mathrm{T}_{-} \mathrm{C}_{-}>\mathrm{T}_{+} \mathrm{C}_{-}$. It is also possible that a negative competitor can direct attention away from a nonnegative target, worsening identification of this target (e.g., $\mathrm{T}_{+} \mathrm{C}_{\mathrm{n}}>\mathrm{T}_{+} \mathrm{C}_{-}$).

If a negative competitor can direct attention and worsen identification of a nonnegative target, why was this phenomenon not observed when comparing $\mathrm{T}_{+} \mathrm{C}_{+}$and $\mathrm{T}_{+} \mathrm{C}_{-}$(i.e., $\left.\mathrm{T}_{+} \mathrm{C}_{+}=\mathrm{T}_{+} \mathrm{C}_{-}\right)$? One possibility may lie in a trend of worse identification for a pair of positive words $\left(\mathrm{T}_{+} \mathrm{C}_{+}\right)$. Unlike with negative words, when a positive word is attended to, it may broaden (rather than concentrate) the attentional focus (Fenske \& Eastwood, 2003), just as an experimentally induced positive emotion can expand spatial attention (Rowe et al., 2007). Possibly, a pair of positive words can dilute attention even more, leaving even fewer resources to be allocated to each of the positive words. Therefore, we suggested that this dilution of attention may cause the similar performance between $\mathrm{T}_{+} \mathrm{C}_{+}$and $\mathrm{T}_{+} \mathrm{C}_{-}$.

\section{Conclusion}

The present study provided empirical evidence suggesting that attention can preferentially be directed to negative words in the presence of competition. We first replicated the results of Zeelenberg et al. (2006), showing that, when only a single Chinese word was presented, emotional words were recognized better than neutral words, but without an identification difference between the positive and negative words. When competition was introduced in Experiment 2, identification accuracy was modulated by the emotional valences of the target and competitor words, where there was a preference in attentional orienting to negative over positive words. In Experiment 3, we ruled out the possibility that the inevitable repetition of stimuli in Experiment 2 led to the preferential difference. Therefore, the present results support our hypothesis that competition induced by multiple stimuli is critical for attentional preferential orienting and the subsequent perceptual enhancement for negative information, though that competition may attenuate when only one of the stimuli has an emotional valence, resulting in a lesser preferential difference. We recommend that future studies apply brain-imaging techniques to understand the neural mechanisms of emotional competition.

Author note This research was supported by grants from Taiwan's Ministry of Science and Technology to S.Y. (MOST 101-2410-H-002083-MY3 and 104-2420-H-002-003-MY2).

\section{References}

Anderson, A. K. (2005). Affective influences on the attentional dynamics supporting awareness. Journal of Experimental Psychology: General, 134, 258-281. doi:10.1037/0096-3445.134.2.258

Arnell, K. M., Killman, K. V., \& Fijavz, D. (2007). Blinded by emotion: Target misses follow attention capture by arousing distractors in RSVP. Emotion, 7, 465-477.

Awh, E., Belopolsky, A. V., \& Theeuwes, J. (2012). Top-down versus bottom-up attentional control: A failed theoretical dichotomy. Trends in Cognitive Sciences, 16, 437-443. doi:10.1016/j.tics. 2012.06.010

Banich, M. T., Milham, M. P., Jacobson, B. L., Webb, A., Wszalek, T., Cohen, N. J., \& Kramer, A. F. (2001). Attentional selection and the processing of task-irrelevant information: Insights from fMRI examinations of the Stroop task. Progress in Brain Research, 134, 459-470.

Bar-Haim, Y., Lamy, D., Pergamin, L., Bakermans-Kranenburg, M. J., \& van IJzendoorn, M. H. (2007). Threat-related attentional bias in anxious and non-anxious individuals: A meta-analytic study. Psychological Bulletin, 133, 1-24. doi:10.1037/0033-2909.133.1.1

Barratt, D., \& Bundesen, C. (2012). Attentional capture by emotional faces is contingent on attentional control settings. Cognition and Emotion, 26, 1223-1237. doi:10.1080/02699931.2011.645279

Bradley, B. P., Mogg, K., \& Lee, S. C. (1997). Attentional biases for negative information in induced and naturally occurring dysphoria. Behaviour Research and Therapy, 35, 911-927.

Buckley, T. C., Blanchard, E. B., \& Hickling, E. J. (2002). Automatic and strategic processing of threat stimuli: A comparison between PTSD, panic disorder, and nonanxiety controls. Cognitive Therapy and Research, 26, 97-115.

Calvo, M. G., \& Castillo, M. D. (2005). Foveal vs. parafoveal attentiongrabbing power of threat related information. Experimental Psychology, 52, 150-162. doi:10.1027/1618-3169.52.2.150

Calvo, M. G., Nummenmaa, L., \& Hyönä, J. (2007). Emotional and neutral scenes in competition: Orienting, efficiency, and identification. Quarterly Journal of Experimental Psychology, 60, 1585-1593. 
Carrasco, M., \& Yeshurun, Y. (2009). Covert attention effects on spatial resolution. In N. Srinivasan (Ed.), Progress in brain research: Vol. 176. Attention (pp. 65-86). Amsterdam: Elsevier.

Cave, K. R., \& Batty, M. J. (2006). From searching for features to searching for threat: Drawing the boundary between preattentive and attentive vision. Visual Cognition, 14, 629-646. doi:10.1080/ 13506280500193107

Dalgleish, T., Taghavi, R., Neshat Doost, H., Moradi, A., Canterbury, R., \& Yule, W. (2003). Patterns of processing bias for emotional information across clinical disorders: A comparison of attention, memory, and prospective cognition in children and adolescents with depression, generalized anxiety, and posttraumatic stress disorder. Journal of Clinical Child and Adolescent Psychology, 32, 10-21.

Desimone, R., \& Duncan, J. (1995). Neural mechanisms of selective visual attention. Annual Review of Neuroscience, 18, 193-222. doi:10.1146/annurev.ne.18.030195.001205

Dijksterhuis, A., \& Aarts, H. (2003). On wildebeests and humans: The preferential detection of negative stimuli. Psychological Science, 14, $14-18$.

Easterbrook, J. A. (1959). The effect of emotion on cue utilization and the organization of behavior. Psychological Review, 66, 183-201. doi: 10.1037/h0047707

Fenske, M. J., \& Eastwood, J. D. (2003). Modulation of focused attention by faces expressing emotion: Evidence from flanker tasks. Emotion, 3, 327-343.

Frischen, A., Eastwood, J. D., \& Smilek, D. (2008). Visual search for faces with emotional expressions. Psychological Bulletin, 134, 662 676. doi:10.1037/0033-2909.134.5.662

Gaillard, R., Del Cul, A., Naccache, L., Vinckier, F., Cohen, L., \& Dehaene, S. (2006). Nonconscious semantic processing of emotional words modulates conscious access. Proceedings of the National Academy of Sciences, 103, 7524-7529. doi:10.1073/pnas. 0600584103

Hahn, S., \& Gronlund, S. D. (2007). Top-down guidance in visual search for facial expressions. Psychonomic Bulletin \& Review, 14, 159165. doi:10.3758/BF03194044

Herd, S. A., Banich, M. T., \& O’Reilly, R. C. (2006). Neural mechanisms of cognitive control: An integrative model of Stroop task performance and fMRI data. Journal of Cognitive Neuroscience, 18, 22 32. doi:10.1162/089892906775250012

Ho, M.-C. (2011). Object-based attention: Sensory enhancement or scanning prioritization? Acta Psychologica, 138, 45-51.

Ho, M.-C., \& Atchley, P. (2009). Perceptual load modulates object-based attention. Journal of Experimental Psychology: Human Perception and Performance, 35, 1661-1669.

Keil, A., \& Ihssen, N. (2004). Identification facilitation for emotionally arousing verbs during the attentional blink. Emotion, 4, 23-35.

LeDoux, J. E. (1996). The emotional brain. New York: Simon \& Schuster.

Lim, S. L., \& Kim, J. H. (2004). Cognitive processing of emotional information in depression, panic, and somatoform disorder. Journal of Abnormal Psychology, 114, 50-61.

Loftus, E. F. (1979). Eyewitness testimony. Cambridge: Harvard University Press.

MacLeod, C., Mathews, A., \& Tata, P. (1986). Attentional bias in emotional disorders. Journal of Abnormal Psychology, 95, 15-20. doi: 10.1037/0021-843X.95.1.15

Mathews, A., \& Mackintosh, B. (1998). A cognitive model of selective processing in anxiety. Cognitive Therapy and Research, 22, $539-560$.

Milham, M. P., Banich, M. T., \& Barad, V. (2003). Competition for priority in processing increases prefrontal cortex's involvement in top-down control: An event-related fMRI study of the Stroop task. Cognitive Brain Research, 17, 212-222.
Mogg, K., Philippot, P., \& Bradley, B. P. (2004). Selective attention to angry faces in clinical social phobia. Journal of Abnormal Psychology, 113, 160-165.

Most, S. B., Smith, S. D., Cooter, A. B., Levy, B. N., \& Zald, D. H. (2007). The naked truth: Positive, arousing distractors impair rapid target perception. Cognition and Emotion, 21, 964-981.

National Languages Committee. (1997). The concise Chinese dictionary word frequency statistics report. Taipei: Ministry of Education.

Norman, D. A., \& Bobrow, D. G. (1975). On data-limited and resourcelimited processes. Cognitive Psychology, 7, 44-64. doi:10.1016/ 0010-0285(75)90004-3

Öhman, A., Lundqvist, D., \& Esteves, F. (2001). The face in the crowd revisited: A threat advantage with schematic stimuli. Journal of Personality and Social Psychology, 80, 381-396. doi:10.1037/ 0022-3514.80.3.381

Palmer, S. E. (1992). Common region: A new principle of perceptual grouping. Cognitive Psychology, 24, 436-447.

Pickel, K. L. (2007). Remembering and identifying menacing perpetrators: Exposure to violence and the weapon focus effect. In R. C. L. Lindsay, D. F. Ross, J. D. Read, \& M. P. Toglia (Eds.), The handbook of eyewitness psychology: Vol. II. Memory for people (pp. 339360). Mahwah: Erlbaum.

Prinzmetal, W., McCool, C., \& Park, S. (2005). Attention: Reaction time and accuracy reveal different mechanisms. Journal of Experimental Psychology: General, 134, 73-92. doi:10.1037/0096-3445.134.1.73

Reynolds, M. G., Eastwood, J. D., Partanen, M., Frischen, A., \& Smilek, D. (2009). Monitoring eye movements while searching for affective faces. Visual Cognition, 17, 318-333.

Rowe, G., Hirsh, J. B., \& Anderson, A. K. (2007). Positive affect increases the breadth of attentional selection. Proceedings of the National Academy of Sciences, 104, 383-388.

Santee, J. L., \& Egeth, H. E. (1982). Do reaction time and accuracy measure the same aspects of letter recognition? Journal of Experimental Psychology: Human Perception and Performance, 8, 489-501. doi:10.1037/0096-1523.8.4.489

Schimmack, U., \& Derryberry, D. E. (2005). Attentional interference effects of emotional pictures: Threat, negativity, or arousal? Emotion, 5, 55-66. doi:10.1037/1528-3542.5.1.55

Schneider, W., Eschman, A., \& Zuccolotto, A. (2002). E-Prime user's guide. Pittsburgh: Psychology Software Tools.

Sørensen, T. A., \& Barratt, D. (2014). Is threat the only modulator of attentional selectivity? Redefining the Easterbrook hypothesis. Frontiers in Psychology, 5, 1021. doi:10.3389/fpsyg.2014.01020

Steblay, N. M. (1992). A meta-analytic review of the weapon focus effect. Law and Human Behavior, 16, 413-424.

van Steenbergen, H., Band, G. P. H., \& Hommel, B. (2011). Threat but not arousal narrows attention: Evidence from pupil dilation and saccade control. Frontiers in Psychology, 2, 281. doi:10.3389/ fpsyg.2011.00281

Vogt, J., De Houwer, J., Koster, E. H. W., Van Damme, S., \& Crombez, G. (2008). Allocation of spatial attention to emotional stimuli depends upon arousal and not valence. Emotion, 8, 880-885.

Weierich, M. R., Treat, T. A., \& Hollingworth, A. (2008). Theories and measurement of visual attentional processing in anxiety. Cognition and Emotion, 22, 985-1018.

Yang, Y. H., \& Yeh, S. L. (2011). Accessing the meaning of invisible words. Consciousness and Cognition, 20, 223-233.

Yantis, S. (1993). Stimulus-driven attentional capture and attentional control settings. Journal of Experimental Psychology: Human Perception and Performance, 19, 676-681. doi:10.1037/00961523.19.3.676

Yeh, S.-L., He, S., \& Cavanagh, P. (2012). Semantic priming from crowded words. Psychological Science, 23, 608-616.

Yeh, S.-L., \& Liao, H.-I. (2008). On the generality of the contingent orienting hypothesis. Acta Psychologica, 129, 157-165. doi:10. 1016/j.actpsy.2008.05.008 
Yeh, S.-L., \& Liao, H.-I. (2010). On the generality of the displaywide contingent orienting hypothesis: Can a visual onset capture attention without top-down control settings for displaywide onset? Acta Psychologica, 135, 159-167.

Yiend, J. (2010). The effects of emotion on attention: A review of attentional processing of emotional information. In J. De Houwer \& D.
Hermans (Eds.), Cognition and emotion: Reviews of current research and theories (pp. 211-275). New York: Psychology Press.

Zeelenberg, R., Wagenmakers, E.-J., \& Rotteveel, M. (2006). The impact of emotion on perception: Bias or enhanced processing? Psychological Science, 17, 287-291. 